\title{
The Least Eigenvalue of the Complement of the Square Power Graph of $G$
}

\author{
Lubna Gul, ${ }^{1}$ Gohar Ali $\mathbb{C}^{1},{ }^{1}$ Usama Waheed, ${ }^{1}$ and Sumiya Nasir $\mathbb{D}^{2}$ \\ ${ }^{1}$ Department of Mathematics, Islamia College, Peshawar, Pakistan \\ ${ }^{2}$ College of Science and Human Studies, Prince Mohammad Bin Fahd University, Khobar Dhahran, Saudi Arabia \\ Correspondence should be addressed to Gohar Ali; gohar.ali@icp.edu.pk
}

Received 28 July 2021; Accepted 2 September 2021; Published 16 September 2021

Academic Editor: Muhammad Javaid

Copyright (c) 2021 Lubna Gul et al. This is an open access article distributed under the Creative Commons Attribution License, which permits unrestricted use, distribution, and reproduction in any medium, provided the original work is properly cited.

Let $G(n, m)$ represent the family of square power graphs of order $n$ and size $m$, obtained from the family of graphs $F(n, k)$ of order $n$ and size $k$, with $m \geq k$. In this paper, we discussed the least eigenvalue of graph $G$ in the family $G(n, m)^{c}$. All graphs considered here are undirected, simple, connected, and not a complete $K_{n}$ for positive integer $n$.

\section{Introduction}

Let $G=(V, E)$ be a simple graph with vertex set $V(G)=$ $\left\{u_{1}, u_{2}, \ldots, u_{n}\right\}$ and edge set $E(G)$. The adjacency matrix of a graph is defined to be the matrix $B(G)=\left[b_{i j}\right]$ of order $n$, where $b_{i j}=1$ if $u_{i}$ is adjacent to $u_{j}$, otherwise $b_{i j}=0$. As the graphs are undirected, $B(G)$ is real and symmetric and the eigenvalues are also real and can be arranged as $\lambda_{1}(G) \leq \lambda_{2}(G) \leq \cdots \leq \lambda_{n}(G)$. The eigenvalues of $B(G)$ are referred to as the eigenvalues of $G$, and one can easily find that $\lambda_{n}$ is the largest absolute eigenvalue among all eigenvalues of $G$.

A graph $G$ is called minimizing in a certain graph class if its least eigenvalue attains the minimum among all the least eigenvalues of the graphs of that class. We will denote the least eigenvalue of a graph $G$ by $\lambda_{\min }(G)$ and $X$ for the corresponding least eigenvector. A graph is said to be a square power graph denoted by $G_{n}^{2}$ of order $n$, if $V\left(G_{n}^{2}\right)=$ $V(G)$ and $E\left(G_{n}^{2}\right)=E(G) \cup\left\{u v: d_{G}(u, v)=2, u, v \in V(G)\right\}$, where $d_{G}(u, v)$ denotes the distance between vertices $u$ and $v$ in $G$; also, the bipartite square power graph is denoted by $G_{n}^{2}(p, q)$, where $p$ and $q$ are the partitions of the vertex set $V\left(G_{n}^{2}\right)$.

We limit our discussion to only connected graphs because we know that the spectrum (the set of eigenvalues of a graph) of a disconnected graph is the union of spectra of its connected components and so the least eigenvalue of a disconnected graph will be the minimum eigenvalue of its component among all the minimum eigenvalues of its components.

There are many results in the literature regarding the least eigenvalue of a graph. Fan et al. [1] discussed the least eigenvalue of complement of trees. Hoffman [2] discussed the limiting point of the least eigenvalue of connected graphs. Hong and Shu [3] discussed the sharp lower bounds of the least eigenvalue of planar graphs. Javid [4, 5] discussed the minimizing graphs of the connected graphs whose complements are bicyclic (with two cycles) and explained the characterization of the minimizing graph of the connected graphs whose complement is bicyclic.

Theorem 1 (see [6]). For a graph $G$ of order $n$,

$$
\lambda_{\min }(G) \geq-\sqrt{\left[\frac{n}{2}\right\rceil\left\lfloor\frac{n}{2}\right\rfloor}
$$

with equality iff $G=K_{\lceil n / 2\rceil,\lfloor n / 2\rfloor}$.

According to Powers et al. [7], if $m$ is the size of a graph $G$, then

$$
\lambda_{\min }(G) \geq-\sqrt{m}
$$


Theorem 2 (see [8]). If G is a graph whose least eigenvalue is minimal among all the connected graphs, then

(i) $G$ is either a bipartite graph, or

(ii) $G$ is a join of two nested split graphs

Theorem 3 (see [9]). Let $K(n, m)$ denote the family of all connected bipartite graphs of order $n$ and size $m$. Let $G \in K(n, m)$ be the graph such that its least eigenvalue is minimal among all the connected bipartite graphs, then $G$ must be a double nested graph.

\section{Main Results}

We begin with some definitions. Given a graph $G$ of order $n$, a vector $X \in R^{n}$ is defined on $G$ if there exists an injective function $f$ from $V(G)$ to the entries of $X$, simply written as $X_{v}=f(v)$ for each $v \in V(G)$. If $X$ is an eigenvector of $B(G)$, then it is naturally defined on $V(G)$ and every entry of $X$ corresponds to the vertex $v \in V(G)$. One can easily prove that

$$
X^{T} B X=2 \sum_{u v \in E(G)} X_{u} X_{v}
$$

and $\lambda$ is an eigenvalue of $G$ corresponding to the eigenvector $X$ if and only if $X \neq 0$, and $N(u)=\{v \in V(G): u v \in E(G)\}$ is called the open neighborhood of $u$ in $G$ :

$$
\lambda X_{u}=\sum_{v \in N(u)} X_{v}, \quad \text { for each vertex } u \in V(G) .
$$

Equation (4) is called the eigen equation for the graph $G$. Now, for any arbitrary unit vector $X \in R^{n}$,

$$
\lambda_{\min }(G) \leq X^{T} B(G) X,
$$

and it will be equal iff $X$ is a least eigenvector of $G$. Now, let $G^{c}$ denote the complement of a graph $G$. One can easily prove that $B\left(G^{C}\right)=J-I-B(G)$, where $I$ and $J$ denote the identity matrix and the ones matrix of the same size as $B(G)$. Now, for any vector $X$,

$$
X^{T} B\left(G^{C}\right) X=X^{T}(J-I) X-X^{T} B(G) X .
$$

A graph $G$ is said to be a tree iff there is a path between any pair of vertices and has no cycle, and a tree is said to be a star iff there exists a vertex of degree $n-1$ and all other vertices have degree one. The former is called the central vertex, and the later is called the pendant vertex. If a graph $G$ is a path of order less than or equal to 4 then $\lambda_{\min }\left(G^{c}\right)=-1$. If a graph $G$ contains $K_{1,2}$ as an induced subgraph, then $\lambda_{\text {min }}(G) \leq \lambda_{\min } K_{1,2}=-\sqrt{2}$. If a graph $G$ is complete or its complement is a null graph or union of disjoint complete graph, then $\lambda_{\min }\left(G^{c}\right)=-1$. So, we are limiting our discussion to $\left(G_{n}^{2}\right)^{c}-K_{n}^{c}$, where $K_{n}{ }^{c}$ denotes all those graphs whose $\lambda_{\text {min }}$ is -1 .

Special tree graph denoted by $G(p, q)$ is obtained from two stars $S_{p-1}$ and $S_{q-1}$ of orders $p$ and $q$, respectively, by joining any two vertices of degree one from the two stars. $G^{2}(p, q)$ is a square power graph of this tree and $G^{2}(p, q)^{c}$ is its complement graph, as shown in Figure 1; the order of $G^{2}(p, q)^{c}$ is $n=p+q$, and its size is $m=p q-3$.

Lemma 1. Given $n \geq 6$, for any positive integers $p$ and $q$ such that $p \geq q \geq 1$ and $p+q=n$,

$$
\lambda_{\min }\left(G_{n}^{2}(p, q)^{c}\right) \geq \lambda_{\min }\left(G_{n}^{2}\left(\left\lceil\frac{(p+q)}{2}\right\rceil,\left\lfloor\frac{(p+q)}{2}\right\rfloor\right)^{c}\right),
$$

with equality iff $p=\lceil(p+q) / 2\rceil$ and $q=\lfloor(p+q) / 2\rfloor$.

Proof. For $n \geq 6$, let $\lambda_{\text {min }}$ be the least eigenvalue of $G_{n}^{2}(p, q)^{c}$ and $X$ be the corresponding least eigenvector. Let $X_{i}$ denote the entries of $X$ corresponding to the vertices of $\left(G_{n}^{2}\right)^{c}$. Now, by eigen equation, all the vertices $u_{1}, u_{2}, u_{3}, \ldots, u_{p-2}$ have the same values, say $X_{1}$ and $X_{2}$ denote the entry of $X$ corresponding to the vertex $u_{p-1}, X_{3}$ for the vertex $u_{p}$, and all the vertices $v_{1}, v_{2}, v_{3}, \ldots, v_{q-2}$ have the same value $X_{4} . X_{5}$ represents the entry of $X$ corresponding to the vertex $v_{q-1}$, and for the vertex $v_{q}$, the entry of $X$ is $X_{6}$. Now, $\lambda_{\min }\left(G_{n}^{2}(p, q)^{c}\right)<-1$. So, by an eigen equation,

$$
\begin{aligned}
& \lambda_{\min } X_{1}=X_{6}+X_{5}+(q-2) X_{4}, \\
& \lambda_{\min } X_{2}=X_{5}+(q-2) X_{4}, \\
& \lambda_{\min } X_{3}=(q-2) X_{4}, \\
& \lambda_{\min } X_{4}=(p-2) X_{1}+X_{2}+X_{3}, \\
& \lambda_{\min } X_{5}=(p-2) X_{1}+X_{2}, \\
& \lambda_{\min } X_{6}=(p-2) X_{1} .
\end{aligned}
$$

Transform (8) into a matrix equation $\left(B-\lambda_{1} I\right) X^{\prime}=0$, where $X^{\prime}=\left(X_{1}, X_{2}, X_{3}, X_{4}, X_{5}, X_{6}\right)^{T}$ and the matrix $B$ of order 6 is omitted. We get

$$
\begin{aligned}
f(\lambda ; p, q)= & \operatorname{det}(B-\lambda I)=(2 p+2 q-p q-4) \\
& -\lambda^{2}(5 p+5 q-3 p q-8)-\lambda^{4}(p q-3)+\lambda^{6} \\
f(\lambda ; p, q)- & f(\lambda ; p-1, q+1)=(p-q-1)\left(\lambda^{4}-3 \lambda^{2}+1\right) .
\end{aligned}
$$

Note that $\lambda_{\min }$ is a least root of $f(\lambda ; p, q)$. In addition, $f(-1,6180 ; p, q)=6.8538-17.9426(p+q)<0$ as $p+q=n$ $\geq 6$, which implies $\lambda_{\text {min }}<-1.6180$.

If $p \geq q$, by the above discussion, for $\lambda_{\min }<-1.6180$, we have

$$
f(\lambda ; p, q)-f(\lambda ; p-1, q+1)<0 .
$$

In particular, $f\left(\lambda_{\min } ; p-1, q+1\right)<0$, which implies

$$
\lambda_{\min }\left(G_{n}^{2}(p, q)^{c}\right) \geq \lambda_{\min }\left(G_{n}^{2}(p-1, q+1)^{c}\right) .
$$

This complete the proof.

Considering the family of square power graphs $G(n, m)$ of order $n$ and size $m$ obtained from the family of graphs $F(n, k)$ of order $n$ and size $k$ with $m \geq k$, we have the following result. 


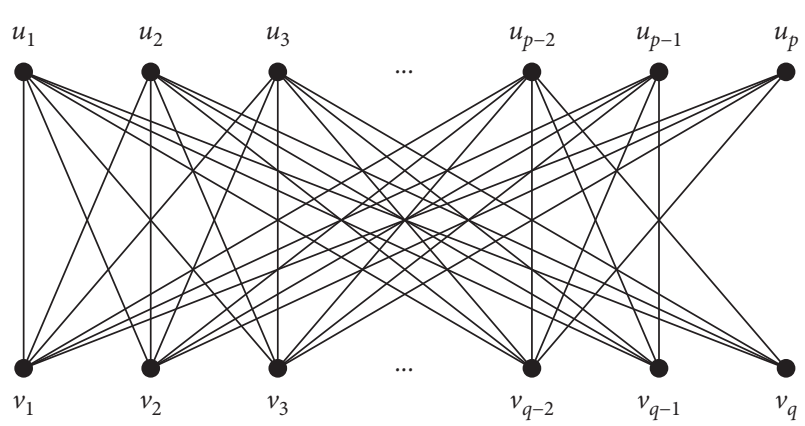

FIgURe 1: $\left(G_{n}^{2}\right)^{c}=G^{2}(p, q)^{c}$ graph with $m=p q-3$.

Lemma 2. Let $G$ be a graph in $G(n, m)^{c}-K_{n}{ }^{c}$ and let $X$ be its least eigenvector, then $X$ contains at least two positive and two negative entries.

Before we state the other results, we need some definitions.

Let $G$ be a bipartite graph with colour classes $U$ and $V$ such that $V(G)=U \cup V ; G$ is said to be a double nested graph if $U=\left\{U_{1} \cup U_{2} \cup \cdots \cup U_{k}\right\}$ and $V=\left\{V_{1} \cup V_{2} \cup \cdots\right.$ $\left.\cup V_{k}\right\}$ such that each $u_{i} \in U_{m}$ is adjacent to every vertex in $V-\cup_{i=k-m+2}^{k} V_{i}$, for $m \in\{1,2,3, \ldots, k\}$.

Now, as we know that, from [9], if two vertices $u$ and $w$ belong to the same colour class and $x_{u} \geq x_{w}$, then $\operatorname{deg}(u) \geq \operatorname{deg}(w)$, where $x_{v}$ is the entry in eigenvector $X$ corresponding to the vertex $v$.

Let $U=\left\{u_{1}, u_{2}, \ldots, u_{k}\right\}$ and $V=\left\{v_{1}, v_{2}, \ldots, v_{k}\right\}$ be the colour classes such that $x_{u_{1}} \geq x_{u_{2}} \geq x_{u_{3}} \geq \cdots \geq x_{u_{k}}$ and $x_{v_{1}} \geq x_{v_{2}} \geq x_{v_{3}} \geq \cdots x_{v_{k}} \geq$, where $x_{u_{i}}$ and $x_{v_{i}}$ for $i \in\{1,2,3, \ldots, k\}$ are the entries of the corresponding eigenvector $X$ of $G$.

Lemma 3 (see [9]). Let $G$ be a graph with the above assumptions, then

(i) The vertices $u_{1}$ and $v_{1}$ are adjacent

(ii) The degree of $u_{1}$ and $v_{1}$ is complete

(iii) If the vertex $u$ is adjacent to $v_{i}$, then $u$ is adjacent to $v_{j}$ for $j<k$, and if the vertex $v$ is adjacent to $u_{i}$, then $v$ is adjacent to $u_{j}$ for $j<k$.

Observation 1. If $G$ is a bipartite graph of order $n$ and size $m$, then $\lambda_{\text {min }}(G)$ goes to its lower bound by increasing the size of $G$.

Bell et al. [9] discussed the behavior of maximum eigenvalue by increasing the number of edges and kept $V(G)$ fixed. We here show the behaviour of least eigenvalue of $G \in G(n, m)^{c}-K_{n}^{c}$ by fixing the number of edges at $m=$ $p q-3$ and increasing the number of vertices. In Figure 2, the horizontal axes show vertices and vertical axes represent least eigenvalues.

Theorem 4. Let $G=\left(G_{n}^{2}\right)^{c}$ be a graph of order $n \geq 6$ from the family $G(n, m)^{c}-K_{n}^{c}$. Then,

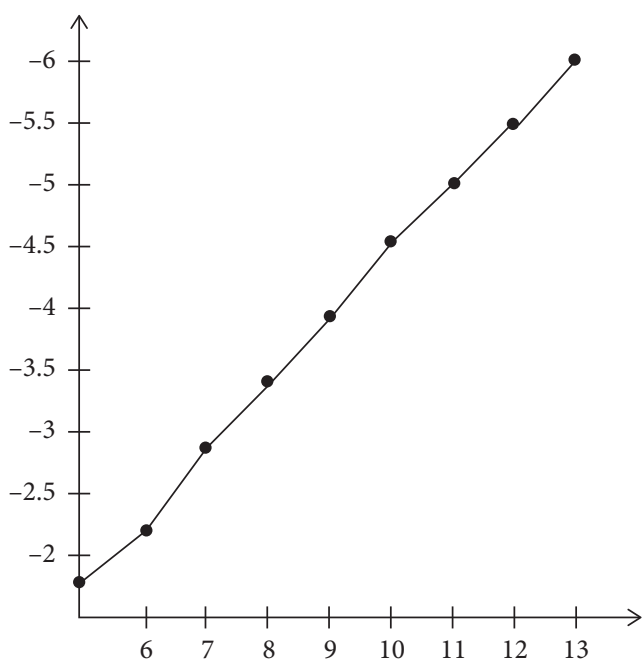

FIgURE 2: Relation between number of vertices and its corresponding least eigenvalue.

$$
\lambda_{\min }\left(\left(G_{n}^{2}\right)^{c}\right) \geq \lambda_{\min }\left(G_{n}^{2}\left(\left\lceil\frac{(p+q)}{2}\right\rceil,\left\lfloor\frac{(p+q)}{2}\right\rfloor\right)^{c}\right) .
$$

Proof. As we know that

$$
\lambda_{\min }\left(\left(G_{n}^{2}(p, q)\right)^{c}\right) \geq \lambda_{\min }\left(G_{n}^{2}\left(\left\lceil\frac{(p+q)}{2}\right\rceil,\left\lfloor\frac{(p+q)}{2}\right\rfloor\right)^{c}\right),
$$

by Theorems 2 and 3 , it is found that $\left(G_{n}^{2}(p, q)^{c}\right)$ is a largest double nested graph. Let $\left(G_{n}^{2}(p, q)^{c}\right)$ be the graph such that $\lambda_{\min }\left(G_{n}^{2}(p, q)^{c}\right)$ is the least one among all the square power graphs and let $X$ be the least eigenvector. Now, let $G_{n}^{2}$ be the graph obtained from $\left(G_{n}^{2}(p, q)^{c}\right)$ by rotating the edge $r_{i s}$ to $r_{i t}$ such that $s \ngtr t$ for $i \in\{1,2, \ldots, k\}$, then $\lambda_{\text {min }} G_{n}^{2} \leq \lambda_{\text {min }}$ $\left(G_{n}^{2}(p, q)^{c}\right)$ and similarly if $s$ and $t$ belong to the same colour class such that $x_{s} \geq x_{t}$, then $\operatorname{deg}(\mathrm{s}) \geq \operatorname{deg}(\mathrm{t})$. So, by Lemma 2.3, it will be a double nested graph. Now, we need to show that it is the largest possible double nested graph. The size of $\left(G_{n}^{2}(p, q)^{c}\right)$ is $p q-3$. So, all we need to show is the size cannot exceed $p q-3$. On the contrary, let $m>(p q-3)$. Then, either $m=p q-2$ or $m=p q-1$. In both cases, the connected square power graph is not possible because if we take $\left(G_{n}^{2}(p, q)^{c}\right)$ with the size greater than $p q-3$, then $\left(G_{n}^{2}(p, q)\right.$ will be a disconnected graph, e.g., $G_{n}^{2}(p, q)$ for $n=8$ which is not allowed. So, this completes the proof [10].

\section{Data Availability}

The data used to support the findings of this study are cited at relevant places within the text as references.

\section{Conflicts of Interest}

The authors declare that they have no conflicts of interest. 


\section{References}

[1] Y. Z. Fan, F. F. Zhang, and Y. Wang, "The least eigenvalue of the complements of trees," Linear Algebra and its Applications, vol. 435, no. 9, pp. 2150-2155, 2011.

[2] A. J. Hoffman, "On limit points of the least eigenvalue of a graph," Ars Combinatoria, vol. 3, pp. 3-14, 1977.

[3] Y. Hong and J.-L. Shu, "Sharp lower bounds of the least eigenvalue of planar graphs," Linear Algebra and Its Applications, vol. 296, no. 1-3, pp. 227-232, 1999.

[4] M. Javaid, "Minimizing graph of the connected graphs whose complements are bicyclic with two cycles," Turkish Journal of Mathematics, vol. 41, no. 6, pp. 1433-1445, 2017.

[5] M. Javaid, "Characterization of the minimizing graph of the connected graphs whose complements are bicyclic," Mathematics, vol. 5, no. 1, pp. 1-12, 2017.

[6] G. Constantine, "Lower bounds on the spectra of symmetric matrices with nonnegative entries," Linear Algebra and Its Applications, vol. 65, pp. 171-178, 1985.

[7] D. L. Powers, "Bounds on graph eigenvalues," Linear Algebra and Its Applications, vol. 117, pp. 1-6, 1989.

[8] F. K. Bell, D. Cvetković, P. Rowlinson, and S. K. Simić, "Graphs for which the least eigenvalue is minimal, I," Linear Algebra and its Applications, vol. 429, no. 1, pp. 234-241, 2008.

[9] F. K. Bell, D. Cvetković, P. Rowlinson, and S. K. Simić, "Graphs for which the least eigenvalue is minimal, II," Linear Algebra and Its Applications, vol. 429, no. 8-9, pp. 2168-2179, 2008.

[10] R. A. Brualdi and A. J. Hoffman, "On the spectral radius of (0, 1)-matrices," Linear Algebra and Its Applications, vol. 65, pp. 133-146, 1985. 